\title{
«Environnement, engagement esthétique et espace public : l'enjeu du paysage »
}

\section{Compte rendu de colloque (Paris, 9-11 mai 2007)}

\author{
Denis Chartier \\ Géographe, Université d'Orléans, Département de géographie, UFR Lettres, Langues et Sciences humaines, laboratoire CEDETE \\ (EA 1210), CREDAL (UMR 7169 CNRS), 10 rue de Tours, BP 46527, 45065 Orléans cedex 2, France
}

Ce colloque était organisé par Nathalie Blanc (géographe, LADYSS, Paris) et Jacques Lolive (politiste, SET, $\mathrm{Pau}$ ). Il s'est tenu dans les locaux de l'École nationale du génie rural, des eaux et des forêts (ENGREF) et a rassemblé 46 intervenants et une soixantaine de participants. Résolument transdisciplinaire, il a réuni anthropologues, philosophes, géographes, urbanistes, architectes, paysagistes, sociologues, historiens de l'art, aménagistes, artistes, sous une même bannière, celle consistant à délimiter les contours de la place de l'esthétique et de l'art dans le domaine de l'environnement. Les trois jours de la rencontre se sont structurés en autant de journées à thème.

La première journée, intitulée "Vers un paysage durable. Du jugement esthétique à l'action publique », a été consacrée à la place de l'esthétique dans les politiques paysagères. Elle a tourné autour de deux ensembles d'interventions. À partir d'études de terrain, le premier a montré l'importance d'un jugement esthétique dans la construction des paysages, ce jugement obéissant à des pratiques et des représentations extrêmement bigarrées. La prise en compte de l'esthétique dans l'action publique s'apparente à une forme d'expérience collective de construction d'une sphère publique facilitant l'acceptation sociale des projets. Le second ensemble regroupait des interventions plus philosophiques et prospectives. Elles proposaient des éléments de théorisation d'une politique de l'environnement qui laisserait une part importante à l' " expérience esthétique ». Cette «intériorisation » de l'environnement est trop souvent négligée, alors qu'elle permettrait de concevoir des politiques dépassant le dualisme nature-culture.

Auteur correspondant : denis.chartier@univ-orleans.fr
Vue ainsi, l'esthétique apparaît comme un point essentiel d'ancrage du paysage dans le vécu de l'existence humaine. L'environnement humain devient environnement humanisé et l'engagement esthétique, le moteur possible d'un renouveau des politiques publiques écologiques - avec ses limites, bien sûr.

La deuxième journée, intitulée "Art et paysage : vers une transformation des écologies locales? », fut consacrée en grande partie à $l^{\prime}$ « art écologique». Elle fut principalement animée par des artistes et des historiens de l'art. Plusieurs interventions ont d'abord présenté les aspects inédits de cet art. Apparu en Amérique du Nord dans les années 1970, il mêle éthique, science et art public. C'est une démarche qui associe souvent artistes, scientifiques et acteurs locaux, un art qui se construit avec l'« esprit des lieux » et dont le but est de résoudre des problèmes plutôt que de les dénoncer. Les approches sont très diverses. Certains artistes ont plutôt une perspective d'interrogation de la démarche artistique; ils sont en quête d'une rénovation des cadres de l'action artistique comme moyen de sensibiliser aux questions environnementales. Cette démarche est aussi apparue comme moteur de négociation, de dialogue avec les scientifiques, avec les habitants, de prise de conscience de la relation à son environnement, d'actions transformatrices sur et dans l'environnement ou l'habitat urbain. Émerge alors un art qui abandonne parfois la voie de la création d'objet et la subjectivité individuelle au profit d'un capital créatif communautaire. D'autres artistes s'intéressent plus à la possibilité de faire vivre une relation au lieu, à un environnement, dans toute sa temporalité. Il s'agit souvent $d^{\prime} œ u v r e s$ paysagères qui témoignent $d u$ passé, des impacts négatifs des activités humaines, tout en laissant ouverte une perspective d'évolution positive, 
en termes écologiques, du site, des consciences et de la relation au lieu. Il s'agit finalement de rendre sensibles des processus auxquels il n'a pas été prêté assez attention (changement climatique, pollutions, etc.), alors qu'ils sont connus, afin de porter un regard critique, constructif et mobilisateur sur la façon de gérer les espaces de vie.

La dernière journée fut consacrée aux «mobilisations environnementales » et au « recadrage de l'expérience esthétique ». Elle est apparue comme une première synthèse. Il s'agissait de savoir, à travers l'analyse d'un certain nombre d'expériences locales, si nous assistions à l'émergence d'une politique des formes. Plusieurs communications ont d'abord prolongé les réflexions de la veille en analysant l'impact d'aménagements paysagers faits sur d'anciens sites industriels, dans des situations où il s'agissait de faire évoluer le lieu sans en effacer la mémoire, de restaurer sans mentir sur le passé, afin d'éviter de contribuer à «la myopie dont souffre l'humanité ». D'autres ont montré que, si un engagement esthétique pouvait permettre de requalifier les lieux d'un point de vue écologique et social, il reste néanmoins difficile, parfois, de "casser » les inégalités d'accès à la nature intrinsèquement liées aux inégalités sociales. Il ressort finalement de cette journée que l'art et l'esthétique peuvent être des outils pour la participation des habitants aux politiques territoriales, des outils susceptibles d'ouvrir sur une démarche très créatrice, moteur d'un renouveau $\mathrm{du}$ politique. En conduisant à repenser notre relation à la ville, en déstabilisant les projets des aménageurs ou en débordant les cloisonnements administratifs et les classifications rassurantes, la prise en compte du monde sensible et l'affirmation de choix esthétiques apparaissent comme le pivot d'une transformation du politique reposant sur une nouvelle relation au monde.

À l'issue de ces trois jours de colloque, il est impossible de ne pas être convaincu de l'originalité et du caractère essentiel des thématiques abordées, bien que, sur le devant de la scène, l'écologie apparaisse trop souvent dans une impasse. Yates McKee parle de «sclérose littérale » qui fait que les acteurs de l'écologie «voient l'environnement comme une chose déterminée qui doit être représentée, protégée et défendue en tant que telle par des experts, plutôt que comme le lieu de médiation d'un réseau de forces sociales, politiques, économiques et naturelles qui concerneraient tous les citoyens ${ }^{1} \gg$. Comment sortir de cette impasse? En gageant, selon le même auteur, que «l'écologie [?] ne sera pas durable tant qu'elle ne s'intéressera pas à l'imagination et à l'identification, ainsi qu'à leurs moyens esthético-culturels » (ibidem). Le colloque valide cette hypothèse en démontrant qu'un engagement esthétique en relation avec les finalités de

1 McKee, Y., 2006. L'art et les fins de l'écologie, Vacarme, 34, 141-147 (http://www.vacarme.eu.org/article547.html). l'écologie peut jouer un rôle significatif dans la réinvention du politique.

Cette réinvention passe par une ouverture aux mondes sensibles. Les exemples présentés pendant cette rencontre montrent que, par des actions de restaurations environnementales temporalisées, par des activités d'échanges autour des connaissances territoriales locales, par la création d'œuvres nécessitant une forte interaction avec les acteurs locaux, les artistes renouvellent les consciences, les relations aux lieux et à l'environnement. « L'artiste fait émerger l'invisible au sein du visible, il inspire l'air rempli de potentialités provenant du cosmos, et son œuvre est expiration ${ }^{2}$ », ce qu'il faut expirer sous peine d'étouffer, disait Jean Cocteau. Cette conception du rôle de l'artiste prend tout son sens dans un contexte de crise écologique. Elle apparaît comme fondamentale dans la mise en place de politiques paysagères et environnementales renouvelées. Insistons sur la dimension de la temporalité : les interventions ont montré qu'elle est essentielle, et trop souvent oubliée dans les politiques menées. Comment reconstruire, comment rénover en témoignant des erreurs passées? Gilles Deleuze disait qu'un monument esthétique ne commémore pas quelque chose qui s'est passé, mais confie à l'oreille de l'avenir les sensations persistantes qui incarnent l'événement ${ }^{3}$. Certains artistes présents dans ce colloque y arrivent. C'est aussi l'une des conclusions de nombreuses communications. Confier à l'oreille de l'avenir les sensations persistantes qui incarnent le présent tout en le réinventant pour éviter les erreurs passées. Voilà ce qu'un engagement esthétique peut apporter, voilà tout l'intérêt de politiques ouvertes aux mondes sensibles.

Il va sans dire que la tâche est lourde et que recherches, expérimentations et réflexions doivent s'affirmer. Il serait souhaitable qu'elles s'inscrivent dans le prolongement des travaux de ce colloque. En initiant la création d'un réseau international associant artistes, scientifiques et acteurs environnementaux, en portant un projet appliqué de Centre d'esthétique et d'écologie, les organisateurs $\mathrm{du}$ colloque s'y emploient. Il faudra mener ces travaux en répondant à quelques manques et/ou défis apparus lors de ces journées. Le premier d'entre eux est la faible place qui y a été accordée aux artistes et aux chercheurs travaillant dans les pays dits du Sud. Au regard de la question traitée (un problème du Nord?), de la nouveauté $\mathrm{du}$ thème pour la communauté scientifique française, cette situation était compréhensible. Il n'en est pas moins indispensable d'inclure ces pays dans la réflexion, tant les relations nature/sociétés et les enjeux urbains y sont différents. Comment parler de l'espace public en ville et de l'esthétique en laissant de côté la question des

\footnotetext{
${ }^{2}$ Brosse, J., 2007. Pourquoi naissons-nous? et autres questions impertinentes, Paris, Albin Michel, p. 159.

3 Cf. Deleuze, G., Guatarri, F., 1991. Qu'est ce que la philosophie?, Paris, Les Éditions de Minuit.
} 
bidonvilles, des quartiers précaires, de la violence sociale induite par le productivisme économique? Comment inscrire cette pensée dans le champ d'une écologie politique à (ré)inventer si l'on se soustrait à ces questions ? Dans un même ordre d'idées, il faut que les recherches à venir ne soient pas «hors sol». Il est apparu que la question de l'esthétique et celle de la justice sociale et environnementale allaient de pair : les recherches à venir devront en tenir compte. Enfin, il faudra veiller à la possible instrumentalisation de l'art. Au cours du colloque, plusieurs intervenants ont mis en garde contre une récupération de l'art, qui annihilerait sa dimension dénonciatrice et novatrice. Il faut aussi veiller à conserver la créativité des artistes, acteurs-clés d'une mise en relation des mondes parallèles.

L'art peut-il ouvrir une sorte de "livre des consciences »? Le colloque ouvre des champs de recherche novateurs et résolument transdisciplinaires qui soulèvent la question et offrent des pistes pour y répondre. Il donne en outre à penser que l'expérience esthétique peut conduire à un nouvel engagement écologique et politique. Il est urgent d'y réfléchir plus avant. Ce champ de recherche, mais aussi d'action, est enfin ouvert. 